\title{
Dialogue inter-tissulaire et modulation de la voie Notch contrôlent l'induction de I'hématopoïèse aortique
}

Cécile Drevon, Charlotte Richard, Aveline Lempereur, Pierre-Yves Canto, Michèle Souyri, Thierry Jaffredo

Hématopoïèse aortique : généralités Chez les embryons de vertébrés, l'aorte est reconnue comme un site majeur d'émergence des cellules souches hématopoïétiques fondatrices du système hématopoïétique adulte [1]. La production hématopoïétique aortique, très conservée chez les vertébrés, est caractérisée par l'apparition de petits bourgeonnements cellulaires qui prennent naissance à partir de l'endothélium du plancher de l'aorte, et qui seront libérés dans la lumière du vaisseau avant d'aller coloniser les organes hématopoïétiques définitifs. L'origine endothéliale de ces bourgeonnements a été clairement démontrée dans différents modèles animaux à l'aide de techniques de traçage cellulaire de l'endothélium et de ses dérivés [2-4], ainsi que par des approches directes de vidéo-cinématographie, soulignant le caractère fortement conservé de cette production [5-7,12]. La formation de ces bourgeonnements passe par la perte progressive des marqueurs endothéliaux et l'acquisition des marqueurs hématopoïétiques. Si les modalités cellulaires de cette émergence hématopoïétique commencent à être comprises, son contrôle moléculaire reste plus énigmatique, et plus particulièrement comment le programme hématopoïétique est induit dans l'endothélium et comment est contrôlée l'expression du facteur de transcription runxl, gène clé de la transition endothélio-hématopoïétique.
Dynamique de l'expression de runx 1 dans l'hématopoìèse aortique

Afin de répondre à cette question nous avons analysé l'expression des facteurs de transcription runxl, c-myb et pu-l, respectivement partenaire et cible de runxl, par hybridation in situ sur des coupes d'embryon de poulet à différents stades de la formation de l'aorte. L'embryon de poulet est un modèle intéressant car son développement procède de manière antéro-postérieure, ce qui permet d'isoler des étapes individualisées dans la formation de l'hématopoïèse aortique. De manière inattendue, runxl et ses partenaires ne sont pas exprimés au cours des premiers stades de formation de l'aorte (Figure IA) mais apparaissent secondairement selon un schéma spatio-temporel strict. L'expression de runxl, suivie quelques heures plus tard par celles de pu-l et myb, apparaît tout d'abord latéralement dans l'endothélium aortique, au stade aortes paires, et ce une journée avant l'apparition de petits bourgeonnements hématopoïétiques appelés clusters aortiques (Figure 1B), puis elle s'étend en direction médiane jusqu'à occuper tout le plancher de l'aorte (Figure IC, D, E). Cette dynamique d'expression de runxl dans l'endothélium suit l'installation du mésenchyme sous aortique (Figure 1). À l'aide d'une technique de traçage cellulaire utilisant des «tatouages» de petits groupes de cellules par des cristaux de carbocyanine Dil, nous avons pu établir que ce mésenchyme était originaire d'une bande de mésoderme
CNRS, UPMC, UMR7622, bâtiment C, $6^{\mathrm{e}}$ étage, case 24, 9, quai Saint-Bernard, 75252 Paris Cedex 05, France.

cecile.drevon@snv.jussieu.fr thierry.jaffredo@upmc.fr

splanchnopleural (ventral) située entre 250 et 300 microns de la ligne médiane de l'embryon. Au-delà de cette distance les cellules mésodermiques marquées se trouvent associées au tube digestif, traduisant une régionalisation médiolatérale de la contribution du mésoderme splanchnopleural. Des résultats similaires ont été obtenus avec des greffes de mésoderme splanchnopleural de caille dans un embryon de poulet hôte [8].

Rôle du mésenchyme sous aortique dans l'initiation de l'hématopoïèse Afin d'analyser fonctionnellement le rôle du mésenchyme sous-aortique sur I'hématopoïèse aortique, nous avons bloqué la mise en place du mésenchyme sous l'aorte à l'aide d'une fente réalisée in vivo sur l'un des côtés de l'embryon (Figure 1A, $A^{\prime}$ ). Cette fente sépare l'embryon proprement dit de la lame latérale; le côté non opéré servant de contrôle. Les embryons ont été observés $24 \mathrm{~h}$ et $48 \mathrm{~h}$ après l'opération. Un jour après l'opération, les aortes paires ont fusionné mais aucun signe moléculaire d'hématopoïèse n'est visible du côté opéré comme en témoigne l'absence d'expression de runxl (Figure 1B', C') alors que du coté non opéré, runxl est exprimé ventralement (Figure IB, C). L'expression de la $V \varepsilon$-cadhérine et de Delta-like 4 (DII4), l'un des ligands de Notch, montre que l'identité vasculaire et artérielle de l'aorte n'est pas perturbée par l'opération. Après 48 heures, un endothélium hématogène épaissi, 


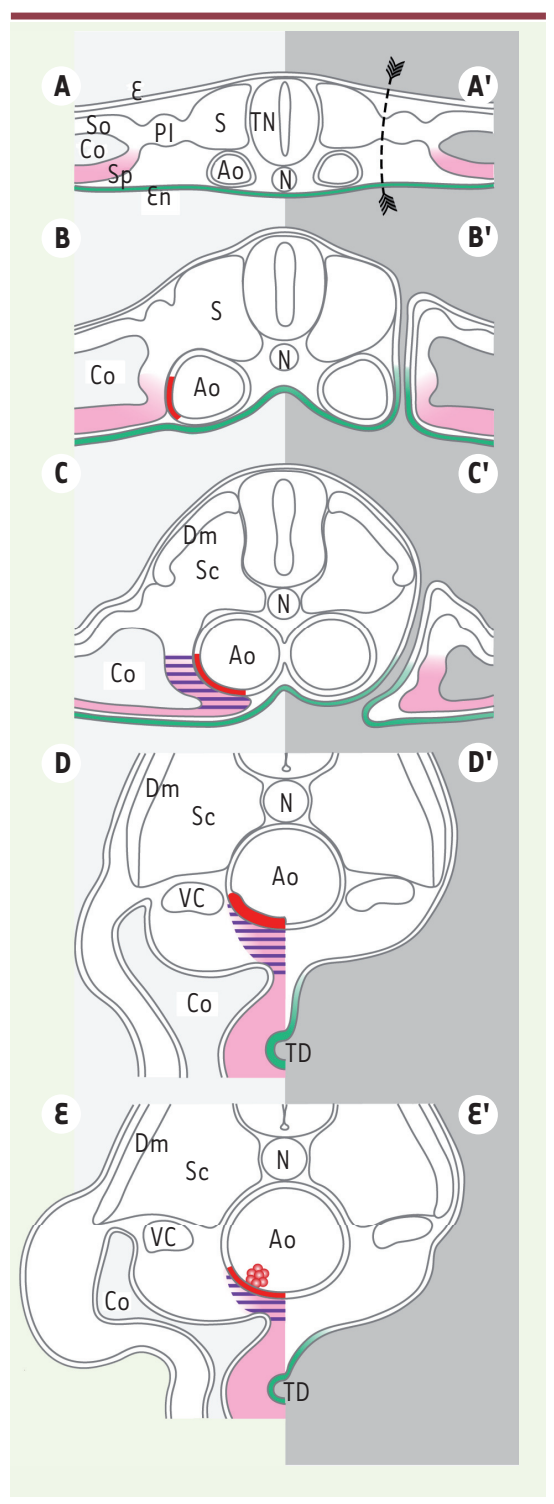

puis des clusters aortiques exprimant runxl, sont visibles du côté contrôle de l'embryon (Figure ID, $\varepsilon$ ), mais pas du côté opéré (Figure 1D', E').

Afin d'éliminer la possibilité que le mésenchyme sous aortique contienne des progéniteurs hématopoïétiques qui puissent contribuer ainsi à la formation des clusters aortiques, nous avons marqué spécifiquement le mésoderme de la lame latérale, mais pas les cellules endothéliales, à l'aide du traceur moléculaire CFDA-SE (carboxyfluorescein diacetate $N$-succinimidyl ester). Cette molécule traverse librement la membrane cellulaire, puis est estérifiée dans le cytoplasme, ce qui contribue à la

Figure 1. Schéma expérimental de la mise en évidence du rôle du mésenchyme sous-aortique dans l'hématopoïèse analysé chez l'oiseau. Le côté contrôle est à gauche (blanc), le côté opéré, à droite (gris foncé). A. L'embryon repose à plat sur le jaune, les aortes sont paires, l'expression de runxl est absente, le mésoderme splanchnopleural est éloigné de l'aorte. A'. La flèche indique le tracé de la coupe qui va séparer le corps de l'embryon de la lame latérale. B. L'embryon commence à se soulever. Le mésoderme splanchnopleural vient au contact de la partie latérale de l'aorte. Suite à ce contact, les cellules endothéliales commencent à exprimer runxl. B'. La lame latérale ne peut pas entrer en contact avec l'aorte. L'expression de runxl n'est pas induite. C. Le mésenchyme sous aortique est en train de se former (rayures violettes). L'expression de runxl s'étend ventralement. C'. Aucune expression de runxl n'est visible du côté opéré. L'endothélium continue d'exprimer les transcrits $V \mathcal{E}$-cadhérine et Delta-like4 attestant du maintien de l'identité artérielle. $D$. L'endothélium exprimant runxl commence à s'épaissir et à exprimer des gènes hématopoiétiques (c-myb, pu-1, CD41). D’. Aucun changement du côté opéré. $\varepsilon$. L'hématopoïèse se poursuit par l'apparition des bourgeonnements intra-aortiques qui expriment runxl, CD4l et CD45. $\varepsilon$ '. Le côté opéré ne montre aucun signe d'hématopoïèse. Ao : aorte ; Co : coelome; Dm : dermomyotome ; $\varepsilon$ : ectoderme ; $\varepsilon$ n: endoderme ; $N$ : notochorde ; S: somite ; Sc: sclérotome ; $\mathrm{PI}$ : pièce intermédiaire ; So : mésoderme somatopleural ; Sp : mésoderme splanchnopleural ; TD : tube digestif ; TN : tube nerveux ; VC : veine cardinale.

séquestrer. Dans ce schéma expérimental, les cellules des clusters aortiques ne sont pas marquées par le CFDA-SE, indiquant que le mésenchyme sous aortique ne contient pas les progéniteurs hématopoḯtiques des clusters aortiques[8]. L'installation du mésenchyme sous aortique apparaît donc comme un commutateur clé responsable d'un dialogue endothélium-mésenchyme qui va induire l'expression de runxl et le programme hématopoïétique dans des cellules endothéliales instruites pour répondre à ce dialogue.

\section{La voie de signalisation Notch et l'hématopoiìse aortique}

La voie de signalisation Notch joue un rôle important au niveau de la communication intercellulaire et des choix binaires de destin cellulaire. Divers travaux ont montré que la voie Notch était requise pour l'hématopoïèse définitive (aortique) chez la souris et le poisson zèbre, et que sa diminution affectait la production hématopoïétique aortique [9-11]. En travaillant sur des stades précis de mise en place de l'hématopoïèse aortique, nous montrons au contraire qu'une diminution de la voie Notch est requise pour la production hématopoïétique aortique. Cette conclusion repose sur un ensemble de données complémentaires: (1) les expressions de runxl et de cjagl et 2, orthologues aviaires des ligands de Notch Jagl et 2 chez les mammifères, sont mutuellement exclusives dans l'endothélium aortique; (2) l'électroporation d'un plasmide rapporteur de l'activité de Notch dans l'aorte d'oiseau montre que l'endothélium aortique et le mésenchyme sous-jacent expriment Notch, mais pas les clusters aortiques; (3) des analyses par PCR quantitative des populations cellulaires issues de l'endothélium hémogénique et des clusters hématopoïétiques de poulet et de souris, triées par cytométrie en flux, indiquent que la signalisation Notch s'éteint au fur et à mesure de la formation des clusters et de la maturation hématopoiétique aortique ; (4) un traitement de 24 heures, soit de l'embryon entier, soit des cultures organotypiques d'aortes par le DAPT, inhibiteur chimique de la voie Notch qui bloque la $\gamma$-secrétase et empêche le clivage du domaine intracellulaire de Notch, augmente dramatiquement le nombre de cellules hématopoïétiques $C D 45^{+}$produites par l'aorte. Des cellules CD45 sont même trouvées au niveau des aortes paires et au niveau du toit de l'aorte, ce qui n'est jamais le cas en situation normale [8]. 


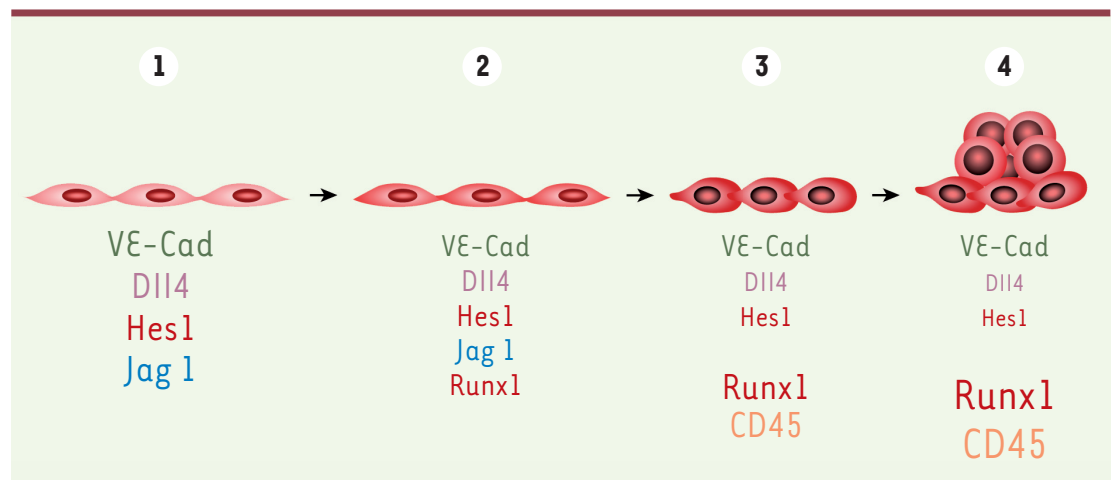

Figure 2. Transitions cellulaires et moléculaires au cours du passage de la cellule endothéliale hématogène vers la cellule hématopoḯtique telles qu'analysées chez l'oiseau et la souris. Le modèle présenté ici résume nos observations concernant l'initiation de l'expression de runxl et la modulation de la voie Notch dans le contexte de l'endothélium aortique. Nos analyses suggèrent que la transition cellule endothéliale-cellule hématopoïétique est caractérisée par au moins quatre stades cellulaires et moléculaires distincts:(1) stade avant l'expression de runxl. Les cellules sont engagées vers la voie hémogénique, mais runxl n’est pas encore présent. Les cellules expriment les molécules VE-cadhérine, DII4, Hesl [hairy enhancer of split] - une cible de la voie Notch -, Jagl. (2) Initiation de l'expression de runxl. Le contact entre le mésoderme splanchnopleural et les cellules endothéliales de l'aorte déclenche l'expression de runxl provoquant la diminution concomitante de plusieurs acteurs de la voie Notch (DII4, Jagl, Hesl) ainsi que celle de la VE-cadhérine. (3) Stade endothélium épaissi. L'expression de runxl se renforce alors que celle les acteurs de la voie Notch diminue (DII4, Hesl) voire s'éteint (Jagl). L'expression de VE-cadhérine est fortement diminuée alors que celle de CD45 est augmentée. (4) Stade clusters hématopoïétiques. L'expression de runxl et de CD45 se renforce. L'expression des différents acteurs de la voie Notch continue de chuter (DII4, Hesl) ou a disparu (Jagl).

\section{Conclusion}

Ainsi, nous avons pu montrer que, chez les vertébrés, l'hématopoïèse aortique est un phénomène très conservé, dont l'initiation via l'expression de runxl est étroitement contrôlée par le mésenchyme sous aortique, et dont la progression est modulée par la voie de signalisation Notch dans l'endothélium hémogénique. Bien que cette signalisation soit largement impliquée dans le processus de formation des vaisseaux et de différenciation veine/artère, nous démontrons que la mise en route du programme hématopoïétique aortique nécessite une diminution de la voie
Notch dans l'endothélium hémogénique puis dans les clusters aortiques. Pour le moment, la nature moléculaire du signal émis par le mésenchyme reste à préciser, mais son identification sera cruciale dans le futur si l'on souhaite, à terme, manipuler des cellules endothéliales dans des buts de production hématopoïétique. $\diamond$

\section{LIEN D'INTÉRÊTS}

Les auteurs déclarent n'avoir aucun lien d'intérêts concernant les données publiées dans cet article.

\section{Tissue to tissue dialogue and modulation of the Notch pathway control aortic haematopoiesis}

LA FONDATION PREMUP : UN OPÉRATEUR DE TERRAIN EN PÉRINATALITÉ RECONNU POUR SON EXCELLENCE \&T SON INTERDISCIPLINARITÉ

La Fondation de coopération scientifique PremUp, unique en Europe, intervient sur la prévention du handicap à la naissance, par la protection de la santé de la femme enceinte et du nouveau-né.

\section{aI $\approx$}

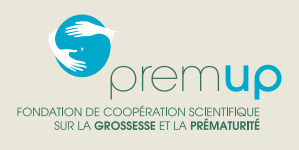

$\stackrel{1}{\circ}$

\section{REMERCIEMENTS}

Les auteurs remercient Sophie Gournet pour son aide précieuse dans les illustrations. Ce travail a été réalisé grâce au soutien du CNRS et de l'UMPC ainsi qu'à l'aide d'une subvention FRM $n^{\circ}$ DEQ20100318258 «Équipe labellisée ». Charlotte Richard a bénéficié d'un financement du ministère de la Recherche et de l'Enseignement supérieur ainsi qu'une bourse de l'ARC.

\section{RéFÉRENCES}

1. Jaffredo T, Richard C, Pouget C, et al. Aortic remodelling during hemogenesis: is the chicken paradigm unique? Int J Dev Biol 2010 ; 54 : 1045-54.

2. de Bruijn M, MaX, Robin C, et al. Hematopoietic stem cells localise to the endothelial cell layer in the midgestation mouse aorta. Immunity $2002 ; 16: 673-83$.

3. Zovein AC, Hofmann JJ, Lynch M, et al. Fate tracing reveals the endothelial origin of hematopoietic stem cells. Cell Stem Cell $2008 ; 3:$ 625-36.

4. Jaffredo T, Gautier R, Eichmann A, Dieterlen-Lievre F. Intraaortic hemopoietic cells are derived from endothelial cells during ontogeny. Development $1998 ; 125: 4575-83$.

5. Boisset JC, van Cappellen W, Andrieu-Soler C, et al. In vivo imaging of haematopoietic cells emerging from the mouse aortic endothelium. Nature 2010 ; 464 : 116-20.

6. Kissa K, Herbomel P. Blood stem cells emerge from aortic endothelium by a novel type of cell transition. Nature 2010 ; 464 : 112-5.

7. Bertrand Jy, Chi NC, Santoso B, et al. Haematopoietic stem cells derive directly from aortic endothelium during development. Nature 2010 ; 464 : 108-11.

8. Richard C, Drevon C, Canto Py, et al. Endotheliomesenchymal interaction controls runxl expression and modulates the notch pathway to initiate aortic hematopoiesis. Dev Cell 2013; 24: 600-11.

9. Bigas A, Espinosa L. Hematopoietic stem cells: to be or Notch to be. Blood 2012; 119: 3226-35.

10. Clements WK, Traver D. Signalling pathways that control vertebrate haematopoietic stem cell specification. Nat Rev Immunol 2013 ; 13 : 336-48.

11. Gering M, Patient R. Notch signalling and haematopoietic stem cell formation during embryogenesis. J Cell Physiol 2010 ; 222 : 11-6.

12. Boisset JC, Robin C. Origine endothéliale des cellules souches hématopoiétiques Med Sci (Paris) 2011 ; 27 875-81.
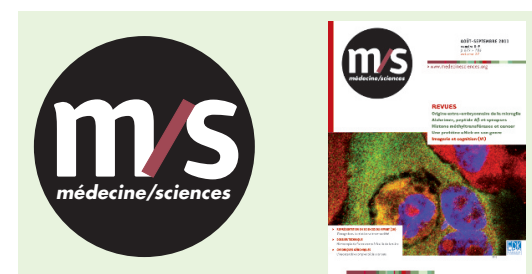

Abonnez-vous

\section{à médecine/sciences}

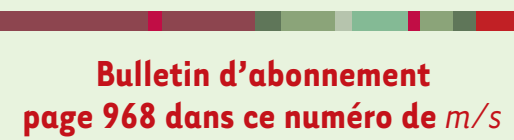

